\title{
Study on the Popper's Scientific Demarcation Criterion
}

\author{
Junheng Sun \\ School of Marxism \\ Wuhan University of Science and Technology \\ Wuhan, China 430065
}

\author{
Bin Wen \\ School of Marxism \\ Wuhan University of Science and Technology \\ Wuhan, China 430065
}

\begin{abstract}
The issue of science demarcation has always been an important issue throughout Western philosophy of science. On the issue of science demarcation, Karl Popper start with critical inductive method and criticized logical positivism, and then puts forward his own standard of falsificationism. His standard of falsificationism is of great importance to the development of science and philosophy, but it faces some difficulties like many other theories.
\end{abstract}

Keywords-Popper; demarcation problem; demarcation criterion; falsificationism

\section{INTRODUCTION}

Karl Popper is a famous Western philosopher of science and social philosopher. He inherits the critical spirit of Einstein of Germany and Kant's rationalism, puts forward the "critical rationalism philosophy", sets up the scientific knowledge perspective opposed to logical positivism and puts forward the knowledge theory of anti-inductivism and falsificationism. In his book Logic of Scientific Discovery, he proposes that there should be a better demarcation criterion between science and metaphysics through criticizing the positivism induction, which is his falsifiable criterion. His falsifiable criterion can be said to have opened up a completely new path for the development of science and philosophy. Most of Popper's later theoretical ideas, including his understanding of scientific knowledge growth, are based on his falsificationism. However, like other scientific theories, Popper's falsification demarcation criterion cannot avoid flaws.

\section{THE ISSUE OF SCIENCE DEMARCATION}

How to distinguish between science and non-science (including pseudo-science)? Is there any criterion for science demarcation? If so, what is the criterion? Popper said: "Finding a standard that allows us to distinguish empirical science from mathematics and logic and 'metaphysical' systems is the demarcation issue I said." The issue of science demarcation is referred as "Kant Question" by Popper. In Kant's mind, this issue became a central issue of epistemology, which is the root of all other issues in epistemology." However, there is no unified view to this focal issue in philosophy of science so far.
There are various understandings on the "demarcation between science and non-science", such as the demarcation between scientific theories and non-scientific theories, the demarcation between scientific methods and non-scientific methods, the demarcation between scientific activities and non-scientific activities, the demarcation of fields of science and non-science, the demarcation of scientific attitudes and non-scientific attitudes, and the demarcation of scientific organizations and non-scientific organizations, etc. That is to say, there can be many explanations to what is the object of demarcation concerning science demarcation? However, the most important and most difficult issue in these scientific demarcations of different meanings is the demarcation problem between scientific theory and non-scientific theory. It is the basis or reference for solving the demarcation problems of scientific and non-scientific of other meanings.

On the issue of science demarcation, Aristotle said in the pre-scientific philosophy period: "Science distinguishes itself from opinions and superstitions through the true reliability of its principles, and distinguishes itself from the craft through its understanding of the first cause. Bacon thinks: "The essence of science is the scientific method." And in the period of logical empiricism, scholars represented by $\mathrm{M}$. Schlick, R. Carnap, H. Reichenbach, A. Ayer, and Hempel proposed the combination of meaning criteria and demarcation criteria, establishing the principle of empirical verification as the demarcation criteria. Logical positivists establish the "principle of empirical verification" of a proposition, which holds that the meaning of a (synthetic or scientific) proposition lies in its verifiability. It lies in that it can be tested by experience, rather than whether it has been confirmed. In short, whether a proposition is meaningful or scientific depends on whether it can be proved. The scientific method used to prove is the "inductive method," and the truthfulness of the inductive principle naturally becomes the precondition and basis for whether inductive methods can be established as empirical verification. From Hume began to realize that inductive principle is facing the problem of its own contradictions. If the inductive principle is regarded as a universal proposition and we try to prove it through experience, it will cause endless retrogression. When Kant settled the issue, he treated the inductive principle as "congenital effective", a principle of universal causality, but he did not adequately address the difficulties. When it comes to Popper, he did not try to solve the problem of inductive 
principle, but directly denied the induction. He thinks the scientific theory or proposition is usually universal proposition, empirical test can only be a singular proposition, and inductive reasoning is to use a limited number of singular propositions to confirm the universal proposition. Then any conclusion drawn in this way can become false. For example, no matter what the examples of white swans we have seen, we can't prove that all the swans are white. Popper uses the fact that "inductive principle" cannot be used to prove if it has not been proved by itself to point out that inductive logic leads either to an infinite retrogression or to transcendentalism and then he proposes his own deductive test, namely falsificationism, as his scientific demarcation criterion.

\section{POPPER'S SCIENTIFIC DEMARCATION CRITERION}

Popper sharply revealed the problem of inductive reasoning in the book "logic of scientific discovery". In his opinion, the universal proposition cannot be derived from singular proposition. Popper cites the typical examples of three inductiveists to contradict the inductive method: (1) the sun rises and falls every 24 hours, which has been the overturned by "frozen sea and midnight sun" discovered by Bitas of Marseille; (2) the claim "biology are destined to die" inferred from "mortal are destined to die has been denied by "bacteria won't die due to self-division; (3) the claim "bread provide human with nutrition" is refuted by ergot poison in the rural areas of France. Therefore, "from the logical point of view, it is clear that it can't be proved that universal proposition inferred from singular proposition (no matter how many of them) is correct because conclusions drawn in this way can always be wrong. Popper said: "The main reason I abandon inductive logic lies in the fact that it does not provide a suitable distinctive emblem that is empirical with systematic theory instead of metaphysics, or that it does not provide a suitable" demarcation criterion." No matter how many times a particular case repeated, it still cannot confirm a universal judgment. Astrology and other nonscientific theory have a large number of accumulated empirical evidence based on observation, fortune-telling map and biography. On this basis, Popper puts forward the "falsifiable criteria". He argues: "what can be used as a demarcation criterion is not verifiability but falsifiability. In other words, I do not require the scientific system to be picked out once and for all in the affirmative sense; I demand it have such logical form: it can be picked out empirically in a negative sense; the scientific system of experience must be able to be refuted by experience. That is to say, the dividing line between science and non-science lies not in whether they can be verified by empirically experience, but whether they can be falsified by experience. Therefore, as long as a theory is falsifiable and refutable, it is scientific; on the other hand, all falsifiable and non-refutable are non-scientific (including not empirical science and pseudoscientific). Popper also pointed out that what he called "falsifiability" in demarcation criterion refers to logically falsifiablity, not whether it has actually been falsified. Falsifiability cannot be equated to having been falsified. Propositions or theories that can be logically falsified are all scientific theories. It includes both the historically falsified theories and the theories that so far have not yet been falsified but which is logically able to be falsified in the future. He thinks any absolutely correct theory or proposition that is logically not falsifiable is nonscientific theory.

Popper, as a critical rationalist, believes that the reason why science is science lies not in whether it is truth or not and whether it can be verified, but whether it is can be wrong, whether it can be falsified and whether it can actively face the contrary evidence. For a proposition, if there is an observation contrary to it, we say that the proposition is falsifiable. The reason why Popper objects to empirical verification and puts forward the empirical principle of falsification was that he finds the asymmetry of the logical relationship between universal proposition and singular proposition. No matter how many white swans you have seen, you cannot confirm the conclusion that "every swan is white." But if you see a black swan, you can falsify it. On this basis, Popper proposes the question of "degree of verifiability". He holds that "some theories are more willing to accept possible refutations than others" and "the more precise one theory is, the more likely it is to be rebutted and the less probability it is, but it is easier to test. Therefore, verifiability will surely increase with the increase of testability". Falsificationism advocates the use of trial and error method and points out that people should boldly put forward hypotheses and guesses, and then look for examples that do not conform to this hypothesis. We can correct hypotheses according to cases, and repeat this process, until the initial hypothesis is rejected in its entirety. The modification and improvement of theory made by trial-anderror method has no limit. The result of trial and error method can only be a better hypothesis, but it is not the best hypothesis. The best hypothesis is the synonym of ultimate truth, which is contrary to the spirit of science. In Popper's view of science, he separates the issue of demarcation from the issue of meaning. Whether there is meaning has no relationship with science and non-science since non-science can also make sense. Popper argues the question of meaning is a pseudo-question and requires excluding "the problem of meaning" as a "pseudo-question." Of course, Popper later made some concessions on this issue. Both logical positivists and Popper agree: Science must have empirical content, while metaphysics has no empirical content. Therefore, both agree that theories need to be tested by according to experience and demarcated based on experience (verifiable or falsifiable). However, "theory should be tested by experience" is only a very crude and general statement. This involves structural problems at the level of experience. It is also related to the problem of relationship between "experience" and "the world". Therefore, we should put forward the question of "truth" and "falsehood" of experience in the ontological sense. It is difficult for this issue to avoid the problem of metaphysics, especially the socalled "realism" issue.

\section{IMPROVEMENT IN POPPER'S SCIENTIFIC DEMARCATION CRITERION}

Popper, in his academic autobiographical book The Infinite Exploration, regards his own resolution of the 
demarcation problem as one of his great discoveries, which he regard as a more important and basic problem than inductive problem. The issue of demarcation is a core part of Popper's philosophy of science. He proposes that the demarcation criterion between science and non-science is the falsifiable principle. This principle is very enlightening and also a breakthrough of people's daily thinking habits. What makes people believe firmly is his theory is based on the valid reasoning form after full conditional reasoning negative consequent. We can see at least the following progress in his falsificationism:

First, it directly points out the defects of verificationism. Popper's demarcation criterion is put forward aiming at logical positivism. It carries out targeted and detailed argument to some errors in logical positivism, which promotes the development of philosophy of science and technology. Logical positivism holds that whether the content of the proposition is scientific lies in whether it can be verified by experience. If yes, the proposition is meaningful and scientific. It is pointless and unscientific if it cannot be verified by experience. In this way, logical positivism separate science from non-science through empirical verification. At the same time, logical positivism holds that the issue of demarcation and the question of meaning are together, so science is meaningful, and nonscience is meaningless. Popper, on the other hand, makes targeted criticism to the viewpoints of logical positivism, including inductive methods, and he believes that any proposition, whether it belongs to the scientific category or not, may have meaning even if it is non-scientific or metaphysical, and inductive reasoning cannot confirm a universal scientific proposition.

Second, he creatively puts forward the method of falsification. This is not just a conversion of thinking angle, but also an innovative test method. Popper captures the characteristics of scientific theories (or propositions) and the constraints of the real world. For a universal proposition, we cannot test it with limited experience, but a limited empirical test can completely negate a universal proposition. This logically asymmetry highlights that falsification and confirmation are not the two sides of a coin. The thinking method of falsificationism is also easy to be accepted by the people's thinking habits, which is of great significance to the development of science. In the history of science, many scientific theories that have been proved wrong inspire people to find out the clue because the counter-examples are found.

Third, he produces a new scientific outlook. Popper's falsification theory essentially denies the absolute truth of scientific theory. Popper believes that any scientific theory can be wrong, and ultimately will be falsified. He stressed that science achieve progress by falsification, and the purpose of science is to constantly approach to the truth and pursue authenticity. We can only reach infinitely get close to the truth, but ultimately cannot reach the absolute truth. Popper pointed out that scientific knowledge is man-made as well as the observation and study of human beings towards nature. At any time, even though at present with relative progress of science and technology advances, human beings have limitations in their observation of nature. This is caused by objective factors such as philosophy, science and technology, and economic level, which is inevitable. Therefore, people may make mistakes when carrying out scientific research and the conclusion may also contain nonscientific statements. However, he does not mean that all scientific theories are wrong. Instead, he tells us that science should be viewed in a dynamic and developmental perspective. Science theory is constantly improved.

Fourth, falsificationism can avoid the defense and doctrine of false theories. If we stick to positivism, once experience that contradicts theory occurs, people can make ad hoc hypothesis or specific limitations that make theories be capable of satisfying the experience. In practice, however, such setting is often extremely unscientific and endless. Just as modern scientists, they increased the number of "epicycle" and "deferent" to reconcile the contradiction between geocentricism systems of Ptolemy and observations. In the end, it just become more and more complicated, and new problems will appear again. Falsificationism convinces people that all science is merely a kind of speculation and hypothesis that will not be verified eventually but will be falsified at any time.

\section{DEFECTS IN POPPER'S DEMARCATION CRITERION}

Popper's demarcation theory is a landmark development in the history of philosophy of science and technology in terms of historical background and the influence of theory. However, we must admit that Popper's theory faces many difficulties, at least the following point:

First, falsifiability can't completely distinguish science from non-science. From the perspective of falsifiability, many theories that have been falsified in the history of science, such as geocentric theory, phlogiston theory, thermo-theory and heliocentric theory, say that oxidation theory and molecular theory are all included in science. And mathematics, logic, metaphysics, pseudoscience, and religious theology are classified as non-scientific, because the propositions in these areas are not falsifiable. The reason why mathematics and logic are divided into non-sciences is that they do not need to be tested by experience. Similarly, psychoanalysis, astrology, and bone science are all nonscientific. None of them can be falsified. In addition, the universal propositions are not falsifiable in nature and are also classified as non-scientific. However, some universal propositions exist in science and cannot be attributed to metaphysical "propositions", such as "There must be life entity similar to human in the universe beyond the earth."

Second, the law of probability cannot be falsified. Popper demands that a scientific theory must indicate in logical structure that it can be falsified by basic statements. However, in fact, Popper's logical falsification cannot be applied to probability problem, because it cannot be falsified in the logic level, and the declarative probabilistic propositions at logical level are correct. Popper holds that all the propositions that contain probabilities and possible relationships are also non-scientific. For example, the propositions such as "Today may be cloudy or sunny," and 
"The hen may or may not lay eggs today." are non-scientific because they logically include all possibility. On probability problem, Popper also sees these problems and proposes to solve it by introducing an agreement, but this contradicts the conventionist view which he has always criticized.

Third, scientific theories can avoid falsification. "Even asymmetry is admitted, it is still impossible for any theoretical system to eventually be falsified for a variety of reasons. For example, introducing ad hoc auxiliary hypothesis, making ad hoc modification for a definition, and even adopting the attitude that simply refuse to recognize any experience can falsify do not create conflicts. It cannot be denied that scientists do not usually do like this. However, it is logically possible to do so. Popper uses "falsifiability" as the demarcation criterion for science and metaphysics, but logically any scientific theory can evade falsification, so he adds an additional methodological requirement: "The Ad Hoc revision is not permitted "to serve as a supplement to the demarcation criterion of falsificationism. But it still seems very inadequate. Popper also took into consideration the complexity of the demarcation criteria, and therefore he claimed that the demarcation criteria of "falsifiability" that he had proposed were merely "a proposal for an agreement or appointment." Popper has repeatedly stressed that his "falsifiability criterion" as a demarcation criterion between science and non-science still need to be complemented by the introduction of his method of identification or empirical science.

Fourth, Popper's "falsificationism" demarcation criterion and his "realism" position are not consistent. He is opposed to conventionism in order to adhere to realism. However, in fact, he cannot persist in his opposition to conventionism. He had explicitly opposed the conventionism, but eventually he himself was forced to recognize: the first is the "agreement" of the methodology. He acknowledged that his "demarcation criterion" was only "a proposal for an agreement and agreement" and the "goal" of science could not be rationally discussed. Therefore, in his sense, the methodology is the discussion only based on the agreement. Second, he acknowledged that accepting the "basic statement" in science is also through the "agreement" because otherwise it will fall into "endless regression." In this way, he was at both ends of the scientific theory - the above is methodology and the lower part is "basic statement" - so they have to introduce the "promise". Third, it must be admitted that the acceptance of the law of probability within the scientific theory is also based on the "agreement". As a result, his opposition to conventionism and basis for "realism" stand is very weak.

\section{CONCLUSION}

In Popper's philosophical thinking, the principle of falsification plays a central role, which is the key for Popper's to solve the "demarcation problem" as well as the basis of Popper's scientific epistemology and methodology. Although the issue of demarcation has not been solved perfectly by Popper's falsificationism, we have seen a new way to solve the problem. The process of establishing a new theory is a new guide to philosophy or science. Perhaps as
Popper said, "Maybe you are right and I am wrong, but only if we work together can we get closer to the truth."

\section{REFERENCES}

[1] Popper. The Logic of Scientific Discovery. Beijing: Science Press, 1986.

[2] Zhou Qi. A Study of Popper's Concept of Scientific Progress. Wuhan University of Science and Technology, 2011.

[3] Zhang Kai. Differentiation and Analysis of Popper 's Demarcation theory, Seeker, 2010.

[4] Lin Dingyi. Popper's theory of demarcation between science and nonscience. Journal of South China University of Technology(Social Science Edition), 2007, 9 (6).

[5] Teng Fang. An Analysis of Popper's view of Falsificationist demarcation. Sun Yatsen University Forum, 2006, 26 (8). 